\title{
Hypofractionated Radiotherapy for Stage I Non-Small Cell Lung Carcinoma in Patients Aged 75 Years and Older
}

\author{
Ajay P. Sandhu ${ }^{1 *}$, Karen Messer ${ }^{2}$, Mark M. Fuster ${ }^{3}$, Lyudmila Bazhenova ${ }^{4}$, Ehatsham Ahmad ${ }^{5}$, Minya \\ $\mathbf{P u}^{6}$, Polly Nobiensky ${ }^{7}$, Stephen Seagren ${ }^{8}$ \\ ${ }^{1}$ UCSD Moores Cancer Center, Radiation Oncology Department, La Jolla, USA; ${ }^{2}$ UCSD Moores Cancer Center, Family \& Preventa- \\ tive Medicine, La Jolla, USA; ${ }^{3}$ VA Medical Center San Diego Dept of Medicine, La Jolla, USA; ${ }^{4}$ UCSD Moores Cancer Center, \\ Medicine, La Jolla, USA; ${ }^{5}$ UCSD Moores Cancer Center, Radiation Oncology Department, La Jolla, USA; ${ }^{6}$ UCSD Moores Cancer \\ Center; ${ }^{7}$ UCSD Moores Cancer Center, Radiation Oncology Department, La Jolla, USA; ${ }^{8}$ UCSD Moores Cancer Center, Radiation \\ Oncology Department, La Jolla, USA. \\ Email: eahmad@ucsd.edu, apsandhu@ucsd.edu,mfuster@ucsd.edu, kmesser@ucsd.edu,mpu@ucsd.edu, pnobiensky@ucsd.edu, \\ lbazhenova@ucsd.edu, sseagren@ucsd.edu
}

Received January $13^{\text {th }}, 2011$; revised April 10 ${ }^{\text {th }}, 2011$; accepted April $19^{\text {th }}, 2011$.

\begin{abstract}
Purpose: We report our single-institution experience using hypofractionated radiotherapy in a patient population 75 years and older diagnosed with stage IA or IB (T1/T2 N0) Non-Small Cell Lung Carcinoma. Materials and methods: This is a single-institution, retrospective analysis examining disease free and overall survival and toxicity after hypofractionated radiation therapy in a patient population 75 years and older diagnosed with stage IA or IB (T1/T2 N0) NSCLC. Between 1991 and 2005, a total of 33 such patients were identified with a median age of 79 years. Patients were treated with a median total dose of 7000 cGy using median daily dose fractions of 250 cGy. Analysis of competing risks (local failure, distal failure or death as the first event) was performed and cumulative incidence functions (CIF) were estimated. Results: The median length of follow-up was 19.8 months (range: 4.3 - 103.8 months). Of the 33 patients treated, 21 (63.6\% of total) had no evidence of disease recurrence on follow-up imaging over the course of the study. Of the 12 patients with disease recurrence, 6 (18.2\% of total) had local failure as the first event and 6 (18.2\% of total) had distant metastasis as the first event. Analysis of competing risks showed that at 5 years, the probability of local failure as the first detected event was 19.5\% (95\%CI: 7.6\%, 35.6\%); the probability of distal failure as the first detected event was 21.5\% (95\%CI: 7.9\%,39.4\%); and the probability of death without recording a failure was $44.1 \%$ (95\%CI: 26.1\%, 60.7\%). There were no treatment related deaths reported. Conclusions: Elderly patients diagnosed with stage I non-small cell lung cancer may safely be offered hypofractionated radiotherapy as an effective option with curative intent.
\end{abstract}

Keywords: Non-small Cell Lung Cancer, Hypofractionated Radiotherapy, Stage I, Older Age

\section{Introduction}

With increased life expectancy, the incidence of lung cancer continues to rise in the older population [1]. Even though surgery is the treatment of choice for stage I Non-small Cell Lung Carcinoma (NSCLC), this option is limited in elderly patients due to concerns for post- operative morbidity and mortality [2-4]. These patients often have a significant burden of co-morbidities, making surgery an option only in few select patients [5]. In addition, long term smoking and consequent poor lung func- tion from associated chronic obstructive lung disease deems a significant proportion of these patients to be non-surgical candidates. Non-intervention and observation as an approach is a poor choice even for elderly inoperable patients due to the significant short-term fatality rate associated with even early-stage untreated NSCLC [6,7]. Radiotherapy is an attractive alternative to surgery in patients with stage I disease who are otherwise not optimal candidates for surgery [8]. We report our single institutional experience utilizing radiotherapy with a hypofractionated approach in patients 75 years of age or older. 


\section{Materials and Methods}

The current report is based on a retrospective analysis examining the outcomes of radiation therapy in a patient population 75 years and older diagnosed with stage IA or IB (T1/T2 N0) NSCLC. The radiation treatment was delivered with a hypofractionated schedule (defined as dose per fraction greater than 200 cGy). All patients were treated at a single institution over the period 1991 - 2005. Patients selected for the analysis met the following inclusion criteria: 1) Age of 75 and above; 2) completion of a course of hypofractionated radiation treatment for NSCLC stage IA or IB (T1 or T2 N0 M0); 3) deemed surgically inoperable due to medical contraindications or surgical refusal; and 4) no restrictions as to location of tumor within the lungs.

\subsection{Clinical Characteristics}

From 1991 to 2005, a total of 33 patients meeting this criterion were included, with 28 deemed to be surgically inoperable and 5 that made the decision for treatment with radiation despite surgical candidacy. The median age of these patients was 79 years (range 75 - 86), and 29 of 33 patients were male (87.9\%); most had stage IA (T1) disease (22/33 or $66.7 \%$; stage IB (T2), $11 / 33$ or $33.3 \%$ ). Table 1 provides a summary of tumor characteristics. Mean tumor size was $26.8 \mathrm{~mm}$ (range: 10 - $50 \mathrm{~mm}$ ). All patients were staged according to the American Joint Commission on Cancer 2002 staging system. The initial evaluation included complete history and physical examination, routine blood tests, pulmonary function tests, chest X-ray, CT scans of chest and upper abdomen, and 18F-fluorodeoxyglucose positron emission tomography (FDG-PET) (in recent years after this modality became available). A tissue diagnosis through biopsy or needle

Table 1. Patient characteristics.

\begin{tabular}{|c|c|}
\hline Median Age (range) & $79(75-86)$ \\
\hline \multicolumn{2}{|c|}{ Gender } \\
\hline Male & 29 (87.9\%) \\
\hline Female & $4(12.1 \%)$ \\
\hline \multicolumn{2}{|c|}{ Tumor Stage } \\
\hline $\mathrm{T} 1$ & $22(66.7 \%)$ \\
\hline $\mathrm{T} 2$ & $11(33.3 \%)$ \\
\hline Mean Tumor Size, mm (range) & $26.8(10,50)$ \\
\hline \multicolumn{2}{|l|}{ Pathology } \\
\hline No Tissue Diagnosis & $5(15.2 \%)$ \\
\hline Squamous & $9(27.3 \%)$ \\
\hline Adeno & $5(15.2 \%)$ \\
\hline NOS & $14(42.4 \%)$ \\
\hline \multicolumn{2}{|c|}{ Dose Per Fraction (cGy) } \\
\hline $220-249$ & $5(15.2 \%)$ \\
\hline $250-400$ & $25(75.7 \%)$ \\
\hline 401-666.7 & $3(9.1 \%)$ \\
\hline
\end{tabular}

aspiration was obtained in 28 (84.8\%) patients, and the remaining $5(15.2 \%)$ patients were treated with a presumptive clinical diagnosis based on imaging results and consensus at the institutional Tumor Board.

\subsection{Treatment Details}

Radiation treatment details (total dose, dose per fraction, frequency, time elapsed over treatment and use of 3-D conformal mapping) were also obtained from medical records. Patients received a median total dose of 7000 cGy using median daily dose fractions of 250 cGy. The total radiation doses used and dose per fraction ranged from 1980 - 7000 cGy and 220 - 666.7 cGy respectively. All patients were treated using hypofractionated schedules with daily dose fractions > 200 cGy, 33/33 (100\%); to be specific, 5 patients received daily dose fractions of 220 - 249 cGy, 25 patients received 250 - 400 cGy, and 3 patients received greater than $400 \mathrm{cGy}$. The most preferred schedule was $250 \mathrm{cGy}$ per fraction to a total dose of 6250 - 7000 cGy in 25 - 28 fractions (63.6\%). The majority of patients $(84.8 \%)$ were treated with the dose fractionation: 220 - 269 cGy in 25 - 30 fractions and total dose ranging between 5000 - 7000 cGy. In a minority of patients with tiny peripheral tumors, shorter fractionation schedules such as 400 cGy in 10 fractions (3\%), or 660 666.7 cGy in 3 fractions (6.1\%), or 257 - 411 cGy in 15 17 fractions (6.1\%) were employed. All patients were treated in a supine position. The majority of patients (after 1995) underwent CT based planning. The gross tumor volume (GTV) was contoured on the free breathing planning scans using lung windows. When PET scanning became available, target information was optimized through fusion with planning CT scans. The clinical target volume was determined by expanding GTV by $1-2$ $\mathrm{cm}$ (more so in superior-inferior than lateral dimensions). When tumors were visible, respiratory motion of the tumor was gauged fluoroscopically for individualized planning margins. There was no attempt to electively treat hilar or mediastinal nodal regions and therefore such regions were not included in the clinical target volume. A single treatment plan was approved to cover the target to prescribed dose and minimizing the dose to normal tissues. The spinal cord was almost always excluded from the radiation portal with few exceptions when dose was kept below 45 Gy depending on dose fractionation employed. Patients were treated with linear accelerator beam energies of 6 - $18 \mathrm{MV}$.

\subsection{Statistical Analysis}

Analysis of competing risks (local failure, distal failure or death as the first event) was performed and cumulative incidence functions (CIF) were estimated. Point estimates of CIFs and their variances at 5 years were calcu- 
lated. Gray's tests were used to assess the association between CIFs and a covariate [9]. Brookmeyer and Crowley's 95\% confidence intervals were calculated for median survival times [10]. Kaplan-Meier curves were plotted for the two survival outcomes of local failure-free survival and overall survival (LFFS and OS). Association between a categorical factor and a survival outcome was assessed using log rank tests. All analyses used the statistical package R version 2.5.1, 2007 (www.r-project.org).

The details of evaluation and analysis of outcomes including local control, survival and toxicity have been outlined in detail in our previous publications [11,12].

\section{Results}

Between 1991 and 2005, 33 patients with inoperable Stage I NSCLC completed a full treatment schedule of hypofractionated radiotherapy with no deaths due to treatment. The median follow-up duration from date of diagnosis to the date of last outcome/follow-up or date of death for this analysis was 19.8 months (range: 4.3 103.8 months).

\subsection{Local Control and Survival}

Of the 33 patients treated, 21 (63.6\%) had no evidence of disease recurrence on follow-up imaging over the course of the study with 6 of the 21 (18.2\% of the 33 total) being event-free and 15 of the 21 (45.5\% of the 33 total) succumbing to death without evidence of disease recurrence. Of the 12 patients with disease recurrence over the course of the study, 6 (18.2\% of the total) had local failure as the first event and $6(18.2 \%$ of the total) had distant metastasis as the first event. Analysis of competing risks (Figure 1) showed that at 5 years, the probability of local failure as the first detected event was 19.5\% (95\%CI: $7.6 \%, 35.6 \%$ ); the probability of distal failure as the first detected event was $21.5 \%$ (95\%CI: 7.9\%, 39.4\%); and the probability of death without recording a failure was $44.1 \%$ (95\%CI: $26.1 \%, 60.7 \%$ ). The event rates and median survival times are listed in Table 2.

Median local failure free survival (LFFS) was 21.2 months (95\% CI: 13.8 - 31.1 months) (Table 2); and median overall survival (OS, graphed in Figure 2) was 21.3 months (95\% CI: 14.4 - 40.7 months). There was not a statistically significant difference in LFFS between the 5 subjects with no tissue diagnosis versus the rest of the subjects (median LFFS time was 21.3 vs. 14.7 months, $p=0.44$ ).

\subsection{Toxicity}

Radiation related toxicity was limited to grade 2 or less and none had grade 3 or higher toxicity. There were no treatment related deaths reported.

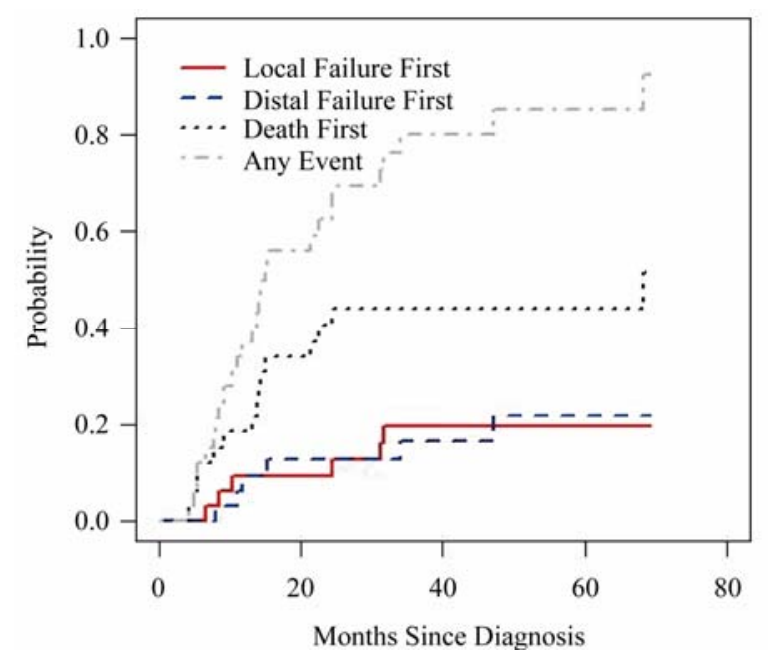

Figure 1. Cumulative incidence functions for competing risk of local failure, distal failure, and death as first observed event. Patients were aged 75 years and older and diagnosed with stage IA or IB (T1/T2 N0) Non-Small Cell Lung Carcinoma between 1993 and 2001, and treated using hypofractionated radiotherapy at UCSD $(n=33)$.

Table 2. Event rates, cumulative incidence functions (CIFs) and median survival times.

\begin{tabular}{|c|c|}
\hline \multicolumn{2}{|c|}{$\begin{array}{l}\text { Number of Events (Estimated Event Rate), CIF (95\%CI) by } 60 \\
\text { Months }\end{array}$} \\
\hline Local Failure First & 6/33 (18.2\%), 19.5\% (7.6\%, 35.6\%) \\
\hline Distal Failure First & 6/33 (18.2\%), 21.5\% (7.9\%, 39.4\%) \\
\hline Death with No Failure & 14/33 (42.4\%), 44.1\% (26.1\%, 60.7\%) \\
\hline Any Event (Death c & 26/33 (78.8\%), 85.1\% (61.7\%, 94.8\%) \\
\hline \multicolumn{2}{|c|}{ Median Survival in Months (95\% CI) } \\
\hline Overall Survival & $21.3(14.4-40.7)$ \\
\hline Local-Failure-Free Survival & $21.2(13.8-31.1)$ \\
\hline
\end{tabular}

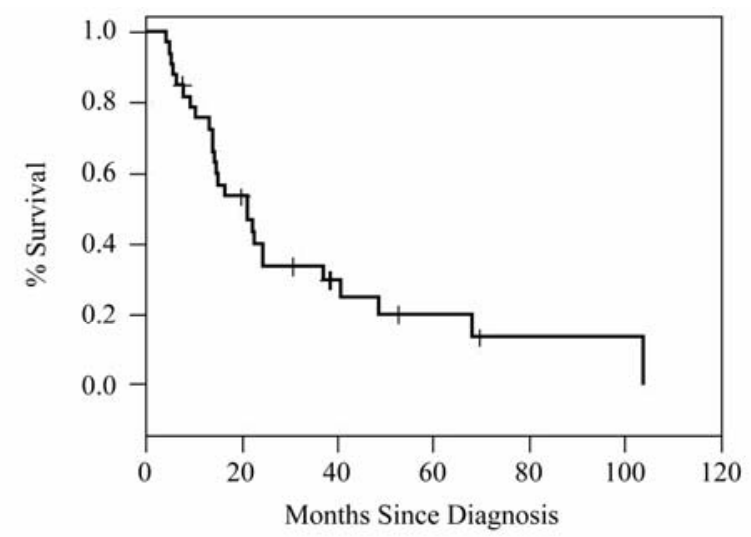

$\begin{array}{llllll}\text { \# at risk: } 33 & 16 & 6 & 3 & 1 & 1 \\ \text { \# deaths: } 0 & 15 & 7 & 2 & 1 & 0\end{array}$

Figure 2. Kaplan-Meier curve showing overall survival. Median overall survival was 21.3 months (95\% CI: 14.40 40.7 months). 


\section{Discussion}

Stereotactic Body Radiation Therapy (SBRT) is currently regarded as the standard treatment for inoperable early stage NSCLC. Prior to implementation of SBRT at our institution, we used conventional techniques to treat stage I NSCLC patients using hypo fractionation schedule. Due to limited data on outcomes of older patients treated with radiotherapy. We report our experience using hypofractionated radiotherapy in patients 75 years of age and older with conformal techniques. We have previously reported our comprehensive experience using conformal radiation planning for stage I lung cancer including hypofractionated radiotherapy [11,12]. This report is based on a subgroup of patients 75 years and older treated with a hypofractionated approach. This is an important issue, as there is wide variation in treatment decision making for this age group with early stage lung cancer. The intervention can vary from radical surgery to no treatment, and there is a paucity of data on outcomes of treatment for elderly patients. This considerable variation in managing elderly patients with lung cancer may be due to multiple reasons, including not only age-related characteristics but also concern among physicians for toxicity and ensuing impact on quality of life for elderly patients undergoing therapeutic interventions. However, some of these concerns may be unfounded due to the lack of good data on outcomes in this age group. The elderly are often underrepresented in clinical trials testing new and aggressive treatment approaches. There is a trend toward under treatment and underutilization of radiotherapy among older patients, and several authors have reported that this may have a negative impact on both the quality and quantity of life, even in elderly patients undergoing conservative management $[6,7,13,14]$.

A significant proportion of patients in their mid 70s and 80 s are not suitable for surgery due to medical co-morbidities, age related frailty, and patient preference. Even patients with potential for resection may opt out of aggressive surgical management due to concerns for morbidity and mortality associated with these procedures. For these patients, radiotherapy remains a viable alternative with good outcomes and minimal toxicity. Our results employing hypofractionated radiotherapy in this age group show impressive local control, with a large proportion being cancer free (or at least without radiographic evidence of progression) at the time of last follow-up or death. Bonfili et al. [8] recently reported similar results using hypofractionated radical radiotherapy in elderly patients with stage I-II non-small cell lung carcinoma. The incidence of both local and distant failure was higher compared to our study possibly due to larger tumor size and inclusion of patients with stage II and N1 disease. In addition, treatment using hypofractionated radiotherapy did not negatively impact quality of life due to minimal associated toxicity as reported by us and other investigators. Even though an earlier study [15] had shown a higher rate of pneumonitis in patients $\geq 70$ years (which was dose dependent) we used a hypofractionated schedule up to 70 Gy with minimal toxicity. This could be attributed not only to modern conformal planning techniques but also to use of involved fields and no attempt to electively treat nodes. Importantly, in spite of tight margins employed, loco-regional failure was low, and estimated to be under $20 \%$ at 5 years.

Some older patients may have a younger biological age; however results are almost always reported based on the chronological age. Therefore treatment decision based on chronological age may hypothetically exclude patients from clinical trials and other appropriate interventions which could effectively prolong their life expectancy and maintain desired quality of life. Selection of patients for conservative treatment based purely on age alone should be discouraged. Our study has limitations in terms of small sample size, single institution experience, and its retrospective nature, thereby limiting the strength of our conclusions and recommendations. Nevertheless, our findings point to the importance of recognizing the safety and potential efficacy of hypofractionated radiation therapy as a modality with curative intent for early-stage NSCLC in this population.

There is an urgent need to design clinical trials specifically for the growing elderly population, especially with a significant and growing degree of lung cancer burden forcing us to seriously focus on outcome and quality of life measures [16]. Optimal treatment recommendations and guidelines in the near-future could be based on evidence from growing prospective and randomized studies, which are currently arbitrary and vague. We also need to develop geriatric oncology services that focus on formulating clinical and research protocols for the elderly cancer population.

\section{REFERENCES}

[1] A. J. Alberg, J. G. Ford and J. M. Samet, "Epidemiology of Lung Cancer: ACCP Evidence-Based Clinical Practice Guidelines (2nd Edition),” Chest, Vol. 132, Supplement 3, 2007, pp. 29S-55S. doi:10.1378/chest.07-1347

[2] W. J. Scott, J. Howington, S. Feigenberg, et al., "Treatment of Non-Small Cell Lung Cancer Stage I and Stage II: ACCP Evidence-Based Clinical Practice Guidelines (2nd Edition)," Chest, Vol. 132, Supplement 3, 2007, pp. 234S-242S. doi:10.1378/chest.07-1378

[3] C. Gridelli, P. Maione and A. Rossi, "Treatment of Stage I-III Non-Small-Cell Lung Cancer in the Elderly,” Oncology, Williston Park, Vol. 20, No. 4, 2006, pp. 373-380. 
[4] A. Dominguez-Ventura, M. S. Allen, S. D. Cassivi, et al., "Lung Cancer in Octogenarians: Factors Affecting Morbidity and Mortality after Pulmonary Resection,” Annals of Thoracic Surgery, Vol. 82, No. 4, 2006, pp. 11751179. doi:10.1016/j.athoracsur.2006.04.052

[5] A. Sandhu and A. J. Mundt, "Radiation Therapy for Older Cancer Patients,” Aging Health, Vol. 2, No. 6, 2006, pp. 919-930. doi:10.2217/1745509X.2.6.919

[6] D. J. Raz, J. A. Zell, S. H. Ou, et al. "Natural History of Stage I Non-Small Cell Lung Cancer: Implications for Early Detection,” Chest, Vol. 32, No. 1, 2007, pp. 11931199.

[7] R. C. McGarry, G. Song, P. des Rosiers, et al., "Observation-Only Management of Early Stage, Medically Inoperable Lung Cancer: Poor Outcome,” Chest, Vol. 121, No. 4, 2002, pp. 1155-1158. doi:10.1378/chest.121.4.1155

[8] P. Bonfili, M. Di Staso, G. L. Gravina, et al., "Hypofractionated Radical Radiotherapy in Elderly Patients with Medically Inoperable Stage I-II Non-Small-Cell Lung Cancer,” Lung Cancer, Vol. 67, No. 1, 2010, pp. 81-85. doi:10.1016/j.lungcan.2009.03.023

[9] M. Pintilie, "Competing Risks: A Practical Perspective," John Wiley \& Sons Ltd., Chichester, 2006.

[10] R. Brookmeyer and J. Crowley "A Confidence Interval for the Median Survival Time,” Biometrics, Vol. 28, 1982, pp. 29-41. doi:10.2307/2530286

[11] A. P. Sandhu, K. Messer, M. M. Fuster, et al., "Definitive
Radiation Therapy for Stage I Non-Small-Cell Lung Carcinoma: Institutional Experience with Contemporary Conformal Planning,” Clinical Lung Cancer, Vol. 10, No. 6, 2009, pp. 433-437. doi:10.3816/CLC.2009.n.081

[12] E. Ahmad, A. Sandhu, M. Fuster, et al., "Hypofractionated Radiotherapy as Definitive Treatment of Stage I Non-Small Cell Lung Cancer in Older Patients,” American Journal of Clinical Oncology, Vol. 34, No. 3, 2010, pp. 254-258. doi:10.1097/COC.0b013e3181dea7a3

[13] I. S. Fentiman, U. Tirelli, S. Monfardini, et al., "Cancer in the Elderly: Why So Badly Treated?” Lancet, Vol. 335, 1990, pp. 1020-1022. doi:10.1016/0140-6736(90)91075-L

[14] N. Bayman, N. Alam and C. Faivre-Finn, "Radiotherapy for Lung Cancer in the Elderly,” Lung Cancer, Vol. 68, No. 2, 2010, pp. 129-136. doi:10.1016/j.lungcan.2009.12.004

[15] K. Koga, S. Kusumoto, K. Watanabe, et al., “Age Factor Relevant to the Development of Radiation Pneumonitis in Radiotherapy of Lung Cancer," International Journal of Radiation Oncology * Biology * Physics, Vol. 14, No. 2, 1988, pp. 367-371. doi:10.1016/0360-3016(88)90445-2

[16] L. Balducci, "Geriatric Oncology: Challenges for the New Century,” European Journal of Cancer, Vol. 36, No. 14, 2000, pp. 1741-1754 doi:10.1016/S0959-8049(00)00169-6 